\section{PHYSICAL CHEMISTRY}

\section{Separating water by spin}

Water molecules have been separated according to the two possible spin orientations of the molecules' hydrogen atoms.

Water is a mixture of two isomers that differ in the direction of their hydrogen spins. The spins are parallel in 'ortho' water and antiparallel in 'para' water. Little is known about the physical and chemical differences between these two isomers because no viable separation method has been available.

Now Gil Alexandrowicz and his co-workers at the Technion-Israel Institute of Technology in Haifa report a procedure in which they shot a slow beam of water molecules through a strong magnetic field. The field acted like a selective lens for ortho water, which, unlike para water, is sensitive to a magnetic field. Purified ortho water was then collected through a small slit.

The work could pave the way for highly sensitive nuclear magnetic resonance experiments, which rely on the alignment of nuclear spins. Science 331, 319-321 (2011)

\section{CANCER BIOLOGY}

\section{Blood vessels'} tumour defence

Tumours rely on the blood vessels that permeate them to survive. However, secretions from the endothelial-cell lining that supports these vessels blunt tumour growth and spread.

Elazer Edelman at the Massachusetts Institute of Technology in Cambridge and his colleagues studied the effect of endothelial cells, which are abundant in tumours, on human breast- and lung-cancer cells. Those grown in media previously occupied by human endothelial cells exhibited a $40 \%$ reduction in growth and were also less invasive. Knocking down a key protein expressed by endothelial cells lowered their ability to block tumour invasion.

Similar anti-tumour effects were seen when the endothelial cells were implanted in mice.

Sci. Transl. Med. 3, 66ra5 (2011) NEUROSCIENCE

\section{Sleep strengthens memories}

Sleep is known to consolidate memories. But what happens to memories if they are reactivated during sleep?

Björn Rasch at the

University of Basel in

Switzerland, Jan Born at the University of Lübeck in Germany, and their colleagues asked 24 volunteers to learn the locations of pairs of matching cards in an array while being presented with an odour. Half of the volunteers then slept for 40 minutes. During this period, some of the sleeping and wakeful volunteers were exposed to the odour again, to reactivate their memories. Next, the volunteers learned a second task designed to interfere with their memories from the first. They were then asked to recall the original locations of the cards.

Those exposed to the odour during sleep had a higher memory-recall rate than did those who were not exposed. Conversely, in awake volunteers reactivation of the memories with the odour increased forgetfulness. Nature Neurosci. doi:10.1038/ nn.2744 (2011)

BIOCHEMISTRY

\section{New microbial metabolism}

Analysis of a microbe thriving in the harsh salty conditions of the Dead Sea has revealed a novel pathway for metabolizing carbon.

Ivan Berg at the University of Freiburg in Germany and his colleagues discovered the methylaspartate cycle by studying the enzymes and intermediates involved in metabolism when Haloarcula

COMMUNITY CHOICE

The most viewed papers in science

\title{
More plants, more cooling
}

\section{AIGHLY READ \\ on www.agu. \\ org the week of \\ 10 January}

Vegetation could offset atmospheric warming by around $0.6^{\circ} \mathrm{C}$ over land during a projected 30 -year period during which atmospheric carbon dioxide levels are double those of pre-industrial times.

Lahouari Bounoua of NASA's Goddard Space Flight Center in Greenbelt, Maryland, and his colleagues ran three long-term climate models simulating various plant feedback effects. These included a response that increases leaf density and decreases photosynthesis at higher $\mathrm{CO}_{2}$ levels. This caused greater water availability and evaporation from plant surfaces - providing additional cooling not previously accounted for. Average global temperatures rose by $1.68^{\circ} \mathrm{C}$, $0.26^{\circ} \mathrm{C}$ less than predicted by conventional models.

The results are in line with observations and suggest that long-term plant responses could moderate global temperatures if $\mathrm{CO}_{2}$ levels are stabilized. Geophys. Res. Lett. doi:10.1029/2010GL045338 (2010)

marismortui is grown on acetate. The pathway converts a molecule called acetyl coenzyme A into the building blocks of sugars, and is only the third such metabolic pathway identified. Among other features, one intermediate of the complex cycle limits the effects of osmosis, helping the microbe to survive in high-salt environments.

The researchers found striking similarities between the enzymes of $H$. marismortui and those found in some ancient bacteria - an indication that the microbe probably acquired components of this pathway by grabbing genes from other species. Science 331, 334-337 (2011) For a longer story on this research, see http://go.nature. com/lbaczj

\section{Fly shows some leg}

Males of a recently identified species of dance fly captured on Mount Fuji in Japan may be using strangely enlarged leg segments to court females.
Christophe Daugeron at the National Museum of Natural History in Paris and his colleagues found that some male specimens of Empis jaschhoforum caught in their traps had normal limbs (pictured below) but roughly an equal number had one forelimb with a huge final segment (top). One fly even had modifications to both forelimbs.

Such a high prevalence of asymmetry in a population with symmetrical features is unprecedented, say the researchers, who ruled out parasites and genetic differences between cells as causes. The engorged limb-tip is probably a sexual signal and the costs associated with this unwieldy feature may explain why slimlegged flies still exist. Biol. Lett. 7, 11-14 (2011)

\section{- NATURE.COM}

For the latest research published by Naturevisit:

www.nature,com/latestresearch 